\title{
SPECTRAL CORRECTION METHOD BASED ON IMPROVED FLAT-TOP CONVOLUTED WINDOW FOR PARAMETER ESTIMATION OF POWER HARMONIC
}

\author{
Shuping Song, Hongzhong Ma, Zhiyan Zhang
}

Original scientific paper Accurate estimation of harmonic parameter is an important task in signal processing of power system. A new class of flat-top windows is proposed, which is generated by self-convolutions of the fast-decaying minimum-sidelobe flat-top (FDMS-FT) window in the time-domain. The mainlobe and sidelobe features of the new window are studied. In addition, to improve the flatness of mainlobe of the new window, the coefficients of its parent window are optimized. A window-length changeable discrete phase difference correction algorithm based on the new window is presented to estimate power harmonic parameter. In order to inspect the efficiency and accuracy of the presented method, several computer simulations and practical experiments were conducted with power multi-frequency signals. Results show that the proposed method can reduce the computation load efficiently and gives a high parameter estimation accuracy of power harmonic.

Keywords: fast Fourier transform; flat-top window; parameter estimation; power system harmonic; spectral correction; spectral leakage

\section{Spektralna korekcijska metoda utemeljena na poboljšanom spiralnom podesivom prozoru za procjenu parametara harmonijske snage}

Izvorni znanstveni članak

Točna procjena harmonijskog parametra je važan zadatak u obradi signala elektroenergetskog sustava. Predlaže se nova vrsta flat-top prozora koja se generira vlastitim konvolucijama brzo padajućeg flat-top prozora (FDMS-FT) u domenu vremena. Proučavaju se značajke bočnog i glavnog režnja novog prozora. Nadalje, kako bi se poboljšala glatkoća glavnog režnja novog prozora, optimiziraju se koeficijenti matičnog prozora. Predstavlja se izmjenjivi algoritam ispravke razlike faze duljine prozora baziran na novom prozoru kako bi se procijenio strujni harmonični parametar. Kako bi se provjerila učinkovitost i točnost prikazane metode, provedeno je nekoliko računalnih simulacija i praktičnih eksperimenata s višefrekvencijskim strujnim signalima. Rezultati pokazuju da predložena metoda može učinkovito smanjiti opseg računanja i daje visok parametar točnosti procjene harmonijske snage.

Ključne riječi: brza Fourierova transformacija; flat-top prozor; harmoničnost elektroenergetskog sustava; procjena parametra; razmazivanje spektra; spektralna korekcija

\section{Introduction}

The use of nonlinear loads is usually the main cause of harmonic distortion in power system [1]. However, due to the operation of distributed generation systems, supply systems also become harmonic sources worsening the harmonic pollution. Harmonic may result in deterioration of power quality and equipment overheats. To suppress harmonic, harmonic parameter should be estimated accurately [2].

Recently, various methods have been presented for the harmonic parameter estimation [3-5]. Among these methods, the fast Fourier transform (FFT) method is the most commonly used technique because of its simplicity and availability in real-time applications [6]. Nevertheless, the FFT is strict with the periodicity of signal and the synchronization of sampling process [7]. When computed from a block of data that is non-periodic, this method suffers from influences known as spectral leakage and picket-fence effect [8]. Owing to the existences of the fundamental frequency offset and inter-harmonic, synchronous sampling cannot be achieved [9], which also cannot be avoided by adjusting the sampling frequency by hardware or software [10]. In practice, the windowed interpolation FFT methods [11, 12], are the effective methods to accurately recover amplitude, phase, and frequency of spectral components for their simple principles and low hardware requirements.

Choosing window with low peak sidelobe level and high sidelobe decaying rate can sufficiently depress the effect of the spectral leakage [13]. There are mainly two approaches to design windows with great sidelobe features. One is adjusting coefficients of classical cosine windows $[14,15]$. The other one is time-convolutions of a specific parent window. For years, several convoluted windows have been presented, including rectangular convoluted window [16], desirable sidelobe window [17], among others. It was verified that convoluted windows have greater side-lobe attenuation and faster decay of side-lobe compared with parent windows, making them more useful for spectral leakage suppression.

The picket fence effect can be eliminated by adopting interpolation algorithms. Up to now, several interpolated algorithms have been presented, for instance, dualspectrum-line interpolation algorithm [18], triplespectrum-line interpolation algorithm [19], and multipoint interpolation algorithm [20].

Comparing with the direct application of the FFT under asynchronous sampling, the windowed interpolated FFT algorithms improve the estimated parameter accuracy of harmonic availably. However, it is at the cost of increasing computational complexity [21]. When simple windows are used for windowing, such as Rectangular window and Hanning window, their interpolated formulas are simple. Unfortunately, the accuracy of harmonic parameter estimation is unsatisfactory due to poor side lobe properties, especially when they are utilized to estimate weak signal components. When complex windows are employed, the accuracy of harmonic analysis is enhanced. Nevertheless, the interpolated formula becomes complicated, and highorder equations should be solved to calculate the value of frequency deviation. Moreover, calculation formula of recovering amplitude is also complicated. To deal with 
these problems, different solving methods have been presented. Salvatore and Trotta presented flat-top window to correct the amplitude error caused by picketfence effect [22]. Yang and Ding gave a window-length changing discrete phase difference correction method to replace interpolation algorithm [23]. However, there still is some lack in these methods. For the traditional flat-top window, the accuracy is not high because of its poor sidelobe and mainlobe performance. When windowlength changing discrete phase difference correction algorithm is applied, the window used for weighting should have excellent sidelobe performance to suppress spectral leakage sufficiently [24]. However, excellent sidelobe performance means the increase of window items and computation burden.

Accordingly, it is necessary to study new harmonic analysis method, which should satisfy the requirements of high speed implementation in embedded systems. The performance of the windowed FFT algorithms is mainly determined by the window function [25]. Consequently, FDMS-FT window [26] is chosen as parent window, and the FDMS-FT convoluted window is subsequently achieved through multiple-time convolution. The new window, with a reasonable order, is of lower peak sidelobe level and higher sidelobe decaying rate compared with classical cosine window, leading to better performance for the suppression of spectral leakage. In addition, to achieve flatter mainlobe of the new window, the coefficients of its parent window are adjusted by optimization procedure. This type of spectrum of new window guarantees high precision in the estimation of harmonic amplitude. Thus, the new window is of flatter mainlobe while its sidelobe performance is retaining. Then, the window-length changeable discrete phase difference correction method with the new flat-top window is presented to estimate harmonic frequency and phase, without using the time-consuming interpolation algorithm. The new method does not need to solve highorder equations. Hence, computation burden is reduced dramatically. Furthermore, the proposed method can get exact solutions under white noise and frequency offset. The implementation via advanced RISC machines (ARM) -based platform verifies the efficiency of the presented method, indicating that it should be a preferable choice for real-time application.

\section{The FDMS-FT convoluted window}

2.1 The FDMS-FT convoluted window under constantlength parent window

FDMS-FT window is one of the combined cosine windows, and its general discrete expression in the time domain is

$$
g(n)=\sum_{m=0}^{M}(-1)^{m} a_{m} \cos \left(\frac{2 \pi}{N} m n\right) \quad n=0,1,2 \ldots N-1
$$

where $a_{m}$ is the window coefficient and subject to the following restriction:

$$
\sum_{m=0}^{M} a_{m}=1
$$

Different FDMS-FT window is defined by using different $M$ and $a_{m}$ settings. The coefficients of FDMS-FT window are listed in Tab. 1.

For a large $N$, the DFT of Eq. (1) can be simplified to the form

$$
G(\lambda)=\frac{\lambda}{\pi a_{0}} \sin (\pi \lambda) e^{-\mathrm{j} \pi \lambda} \sum_{m=0}^{M}(-1)^{m} \frac{a_{m}}{\lambda^{2}-m^{2}}
$$

in Eq. (3), $\lambda$ is the normalized frequency (normalized by the frequency resolution), and $G(\lambda)$ is normalized by its central frequency bin $G(0)$.

There are two ways for constructing self-convolution window, including constant-length parent window and constant-length convoluted window. Similarly, following the similar approach of convoluting parent window in [16, 17], the new window is achieved

$g_{C}(n)=\underbrace{g(n) * g(n) * \cdots g(n)}_{C}$

where $C$ is the number of the parent window, or called the order of the new flat-top window. Accordingly, the new window $g_{C}(n)$ names the $C^{\text {th }}$-order FDMS-FT convoluted window. It can be seen that the length of $g_{C}(n)$ is $N \cdot C-(C-1)$. Zero padding is performed on $g_{C}(n)$, making it has the length of $N \cdot C$.

In the light of the convolution theorem in the frequency domain, the DFT of $g_{C}(n)$ is

$G_{C}(\lambda)=\left[\frac{\lambda}{\pi a_{0}} \sin (\pi \lambda) e^{-\mathrm{j} \pi \lambda} \sum_{m=0}^{M}(-1)^{m} \frac{a_{m}}{\lambda^{2}-m^{2}}\right]^{C}$

From Eq. (5), it can be seen that the peak sidelobe level of $G(\lambda)$ is $C$ times lower than that of its parent window. By expanding the term $\left(\lambda^{2}-m^{2}\right)^{-1}$ in a power series in $(m / \lambda)^{2}$, Eq. (5) can be rearranged as

$G_{C}(\lambda)=\left[\frac{\lambda \sin (\pi \lambda)}{\pi a_{0}} e^{-\mathrm{j} \pi \lambda} \sum_{r=0}^{\infty} \frac{1}{\lambda^{2 r}} \sum_{m=0}^{M}(-1)^{m} k^{2 r} a_{m}\right]^{C}$

The sidelobe decaying rate of $G(\lambda)$ depends on the first nonzero term in $r$-series [15]. Thus, according to Eq. (6), the sidelobe decaying rate of $G_{C}(\lambda)$ becomes $C$ times greater than that of $G(\lambda)$.

When $C$ equals 1,2 , and 3 , respectively, the magnitude frequency responses of $G_{C}(\lambda)$, are shown in Fig. 1, where 5-term fast-decaying minimum-sidelobe flat-top window (FDMS-5FT) is taken as parent window. One can see that the peak sidelobe level and the sidelobe decaying rate of $G_{C}(\lambda)$ are proportional to the order $C$. 


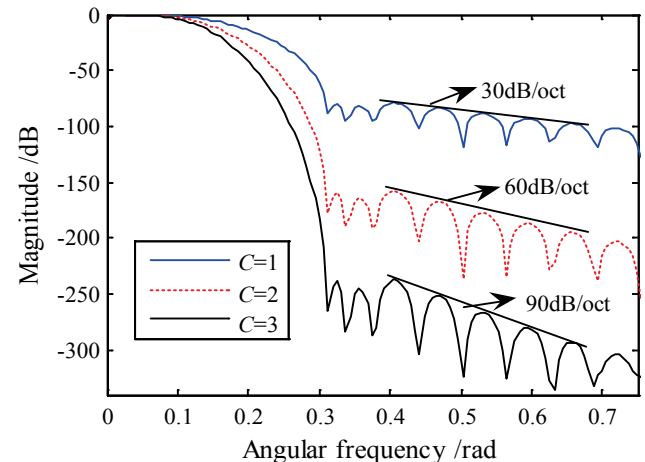

Figure 1 Magnitude frequency responses of the FDMS-5FT convoluted window with constant-length parent window

To describe the flat degree of mainlobe of $G_{C}(\lambda)$, a percentage error $\tau$, which is characterized by constant DFT magnitude around its central frequency bin, is introduced. The expression of $\tau$ can be written as

$\tau=100 \times\left[G(\lambda)^{C}-1\right]$

For simplicity, $\tau$ is named as the attenuation error of mainlobe. Because of the even symmetry of $G_{C}(\lambda), \tau$ is only discussed where $\lambda$ is in the $[0 ; 0,5]$ interval. The curves of $\tau$ with different convoluted order are exhibited in Fig. 2.

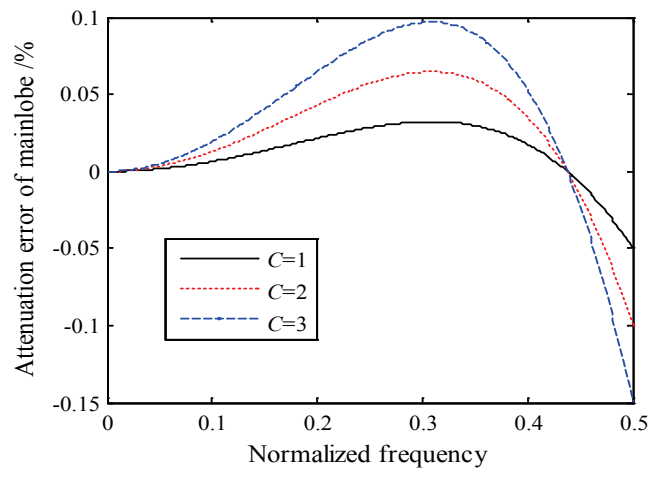

Figure 2 The attenuation error of mainlobe of the FDMS-5FT convoluted window with constant-length parent window

It can be recognized that $\tau$ becomes large with the increase of convoluted order, leading to the decrease of the flatness of mainlobe.

\subsection{The FDMS-FT convoluted window with a fixed window length}

Assuming that the length of the $P^{\text {th }}$-order FDMS-FT convoluted window $s_{p p}(n)$ with a constant-length is $N_{P P}$, thus, the length of its parent window $s_{1 P}(n)$ is

$N_{1 P}=\left(N_{P P}+P-1\right) / P$

Since $N_{P P} \square P \geq 1$, the length of $s_{1 P}(n)$ is approximately $\mathrm{P}$ times shorter than that of $s_{p p}(n)$ (i.e., $N_{1 P}=N_{P P} / P$ ).

From Eq. (3), the DFT of $s_{1 P}(n)$ is

$$
S_{1 P}(\lambda)=\frac{N_{1 P}(\lambda / P)}{\pi} \sin \left(\frac{\pi \lambda}{P}\right) e^{\frac{-j \pi \lambda}{P}} \sum_{m=0}^{M}(-1)^{m} \frac{a_{m}}{(\lambda / P)^{2}-m^{2}}
$$

According to Eq. (9) and the convolution theorem of frequency domain, the DFT of $s_{p p}(n)$ can be expressed as

$$
S_{P P}(\lambda)=\left[S_{1 P}(\lambda)\right]^{P}
$$

Referring to Eq. (10), we know that the sidelobe attenuation and asymptotic behavior of $S_{P P}(\lambda)$ are $P$ times greater than those of $S_{1 P}(\lambda)$. The magnitude frequency responses of $S_{P P}(\lambda)$, where the convoluted order $P$ amounts to 1, 2, and 3, respectively, are shown in Fig. 3.

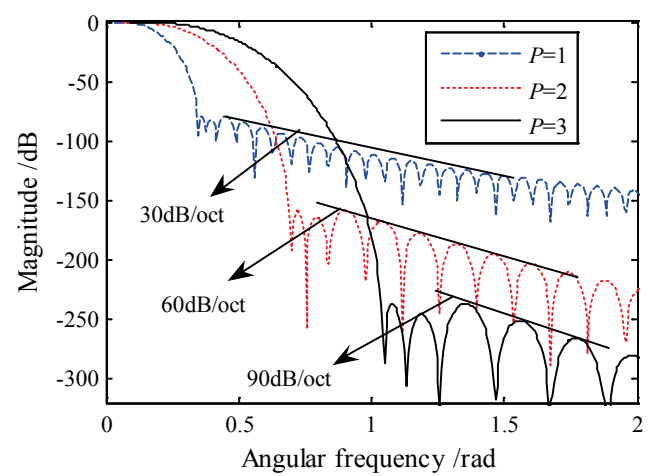

Figure 3 Magnitude frequency responses of the FDMS-5FT convoluted window with constant length

According to Fig. 3, it is clearly indicated that, with the increase of the convoluted order, the peak sidelobe level decreases and the sidelobe decaying rate increases.

In order to describe the flat degree of mainlobe of the FDMS-FT convoluted window with a fixed length, the attenuation error of mainlobe $\delta$ is also introduced. Likewise, $\delta$ is also analyzed in $[0 ; 0,5]$ interval of normalized frequency. Fig. 4 illustrates the curves of $\delta$ with different convoluted order. It is shown that the mainlobe of $S_{P P}(\lambda)$ is flatter than that of $S_{1 P}(\lambda)$ when $\lambda$ is in the range of $[0 ; 0,37]$. However, this has changed with the increase of $\lambda$.

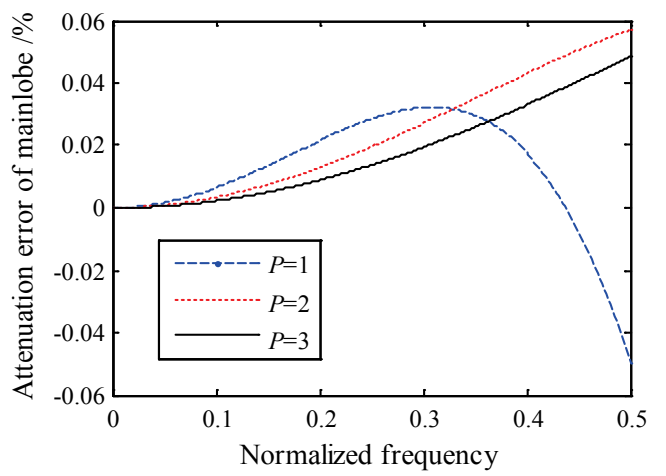

Figure 4 The attenuation error of mainlobe of the FDMS-5FT convoluted window with a constant length

To sum up, from Fig. 1 to 4 , it is summarized that, compared with their parent windows, the two types of FDMS-FT convoluted windows have better sidelobe 
behaviors. However, the flat degrees of their mainlobes are not superior to those of their parent windows.

\section{The improved FDMS-FT convoluted window with a constant length}

From the study in section 2, we know that the two types of FDMS-FT convoluted windows are unfulfilling on the flatness of mainlobe. Therefore, it is necessary to improve their flatness of the mainlobe for the sake of utilization in harmonic amplitude estimation. The FDMSFT convoluted window with constant-length parent window is a multiple of its parent window in the frequency domain, so that its mainlobe will never be flatter than that of parent window. Thus, we choose to enhance the flatness of mainlobe of the FDMS-FT convoluted window with a constant length by optimizing the coefficients of its parent window.

Without loss of generality, the optimization procedure of the coefficients of parent window satisfies the normalized constraint Eq. (2).

The influences among distant harmonic components are determined by the sidelobe decaying rate of window. Thus, expanding $\left[(\lambda / P)^{2}-h^{2}\right]^{-1}$ in a power series in $(h P / \lambda)^{2}$ in Eq. (9), the following expression is obtained

$$
S_{1 P}(\lambda)=\frac{N_{1 P} P}{\pi \lambda} \sin \left(\frac{\pi \lambda}{P}\right) e^{\frac{-\mathrm{j} \pi \lambda}{P}} \sum_{q=0}^{\infty} \frac{1}{\lambda^{2 q}} \sum_{m=0}^{M}(-1)^{m} a_{m}(P m)^{2 q}
$$

Since the sidelobe decaying rate of $S_{1 P}(\lambda)$ depends on the first nonzero term of $q$-series, the coefficients of $S_{1 P}$ satisfy

$$
\begin{aligned}
& \sum_{m=0}^{M}(-1)^{m} a_{m}=0 \\
& \sum_{m=0}^{M}(-1)^{m} a_{m} m^{2}=0
\end{aligned}
$$

The influences among adjacent harmonic components can be reduced by decreasing the peak sidelobe level of window. A low peak sidelobe level is achieved by adding additional zeros between existing zeros of the DFT window's spectrum [15]. Accordingly, to reduce the peak sidelobe level of $s_{1 P}$, the following condition should be satisfied

$$
\sum_{m=0}^{M}(-1)^{m} \frac{a_{m}}{\lambda_{1 P}^{2}-m^{2}}=0
$$

where $\lambda_{1 P}$ is the position placing additional zero.

Specially, in order to enhance the flat degree of mainlobe of the parent window, the attenuation error of mainlobe $\delta$ should amount to zero. Thus, the following constraint is obtained

$$
\sum_{m=0}^{M}(-1)^{m} \frac{a_{m}}{\left(\lambda_{P}^{\prime} / P\right)^{2}-m^{2}}=\frac{\pi a_{0}}{\sin \left(\frac{\pi \lambda_{P}^{\prime}}{P}\right)\left(\lambda_{P}^{\prime} / P\right)}
$$

where $\lambda_{P}^{\prime} / P$ is the zero crossing point of the attenuation error of mainlobe.

By using normalization condition Eq. (2) and constraints Eq. (12) to Eq. (15), the optimized coefficients of FDMS-FT window can be obtained. Then, the new FDMS-FT convoluted window with a fixed length, which has a flatter mainlobe, is achieved through time convolution. Table I lists the optimized coefficients of $s_{1 P}$, where the corresponding order of the new FDMS-FT convoluted window with a fixed length is the second to the fourth. For clarity, the new window is called the improved FDMS-FT convoluted window.

The improved FDMS-5FT convoluted window is taken for example, and the attenuation error of its mainlobe $\varepsilon$ is shown in Fig. 5. In Fig. 5, the order of convolution $F$ amounts to 1,2 , and 3 , respectively.



Figure 5 The attenuation errors of mainlobe of the improved FDMS5FT convoluted window

It can be seen that the flatness of mainlobe of the improved FDMS-FT convoluted window is greatly increased compared with Fig. 4. The higher the convoluted order of the improved FDMS-FT convoluted window is, the lower the attenuation errors of the mainlobe will be.

Accordingly, compared with classical flat-top window in $[22,26]$, the improved FDMS-FT convoluted window exhibits both flatter mainlobes and greater sidelobe performance. Thus, the new window can suppress spectral leakage and enhance the accuracy of harmonic amplitude more effectively.

\section{Harmonic parameter estimation based on the improved FDMS-FT convoluted window}

\subsection{Estimation of harmonic amplitude}

For a sampled multi-frequency harmonic signal in power system, the discrete-time signal model can be written as

$$
x(n)=\sum_{k=1}^{K} A_{k h} \cos \left(2 \pi k n f_{0} T_{s}+\phi_{k h}\right), n=0,1, \ldots, N-1
$$


where $K$ is the number of frequency components, $f_{0}$ is the fundamental frequency, $A_{k h}$ and $\phi_{k h}$ is the amplitude and initial phase of the $k$ th harmonic, respectively. $T_{s}$ is the sampling period.

Table 1 Parent window coefficients of the improved FDMS-FT convoluted window

\begin{tabular}{|c|c|c|c|c|c|c|}
\hline \multirow{2}{*}{ Window } & \multirow{2}{*}{ The order of convolution } & \multicolumn{5}{|c|}{ The coefficients of parent window } \\
\hline & & $\mathrm{a}_{0}$ & $\mathrm{a}_{1}$ & $\mathrm{a}_{2}$ & $\mathrm{a}_{3}$ & $\mathrm{a}_{4}$ \\
\hline \multirow{4}{*}{ FDMS-4FT } & 1 & 0,2328617 & 0,4469165 & 0,2671383 & 0,0530835 & \\
\hline & 2 & 0,2348246 & 0,4474600 & 0,2651750 & 0,0525360 & \\
\hline & 3 & 0,235196 & 0,447586 & 0,264804 & 0,052414 & \\
\hline & 4 & 0,2353258 & 0,4476286 & 0,2646742 & 0,0523714 & \\
\hline \multirow{4}{*}{ FDMS-5FT } & 1 & 0,2014249 & 0,3929181 & 0,2850455 & 0,1070819 & 0,0135296 \\
\hline & 2 & 0,2026808 & 0,3937519 & 0,2839268 & 0,1062481 & 0,0133924 \\
\hline & 3 & 0,2029153 & 0,3939063 & 0,2837171 & 0,1060937 & 0,0133676 \\
\hline & 4 & 0,20298965 & 0,39395525 & 0,28365063 & 0,10604475 & 0,01335972 \\
\hline
\end{tabular}

Under asynchronous sampling, $x(n)$ is weighted by the improved FDMS-FT convoluted window $w_{L}(n)$ with a length $N$. Subsequently, $x_{N}(n)$ is obtained. Suppose that the $k$ th harmonics is currently under concern. The FFT of the $k$ th harmonics is given as follows:

$$
\begin{aligned}
& X\left(\gamma_{k}\right)=\frac{A_{k h}}{2} \sum_{k=1}^{I} W_{L}\left(\gamma_{k}-\gamma_{k h}\right) e^{-\mathrm{j}\left[\pi\left(\gamma_{k}-\gamma_{k h}\right)-\phi_{k h}\right]} \\
& \left.+W_{L}\left(\gamma_{k}+\gamma_{k h}\right) e^{-\mathrm{j}\left[\pi\left(\gamma_{k}+\gamma_{k h}\right)+\phi_{k h}\right]}\right]
\end{aligned}
$$

In Eq. (17), $\gamma_{k h}$ and $\gamma_{q h}$ are the normalized frequency (normalized by frequency interval $F_{0}=1 / N T_{s}$ ) of the $k^{\text {th }}$ and $q^{\text {th }}$ harmonic, respectively. Due to asynchronous sampling, $\gamma_{k h}$ is not an integral multiple of frequency bin Accordingly, $\gamma_{k}-\gamma_{k h} \neq 0$, and $\left|\gamma_{k}-\gamma_{k h}\right|<0,5$. In this case, $X\left(\gamma_{k}\right)$ is the maximum spectral line corresponding to the $k^{\text {th }}$ harmonics.

Because of the excellent sidelobe behaviors of the window $w_{L}(n)$, the leakage impact of other harmonic on the $k^{\text {th }}$ harmonic can be neglected. Thus, Eq. (17) can be simplified as

$$
X\left(\gamma_{k}\right)=\frac{A_{k h}}{2} W_{L}\left(\Delta \varsigma_{k}\right) e^{-j\left(\pi \Delta \varsigma_{k}-\phi_{k h}\right)}
$$

where $\Delta \zeta_{k}$ is the value of frequency deviation of the $k$ th harmonic, and $\Delta \varsigma_{k}=\gamma_{k}-\gamma_{k h}$.

The improved FDMS-FT convoluted window has an extremely flat-top mainlobe. For example, in the case that the value of frequency deviation $\Delta \varsigma_{i}$ is fluctuating in the range $[-0,5 ; 0,5]$, the maximum attenuation error of mainlobe, in which the improved $2^{\text {nd }}$-order FDMS-FT convoluted window is considered, is only $0,0039 \%$. Accordingly, the amplitude $A_{i h}$ of the $k^{\text {th }}$ harmonic can be substituted by $2\left|X\left(\gamma_{k}\right)\right|$ directly. Thus

$$
A_{k h}=2\left|X\left(\gamma_{k}\right)\right|
$$

\subsection{Estimation of harmonic frequency and phase}

The window-length changeable discrete phase difference correction algorithm is applied to estimate harmonic frequency and initial phase.

From Eq. (18), the phase relationship between the $k$ th harmonic and the spectral line $X\left(\gamma_{k}\right)$ is

$\phi_{k h}=\varphi_{\gamma_{k}}+\pi \Delta \varsigma_{i}$

where $\varphi_{\gamma_{k}}$ is the phase of the spectral line $X\left(\gamma_{k}\right)$. According to Eq. (20), it is seen that $\Delta \varsigma_{k}$ is the key factor for the estimation of $\phi_{k h}$.

After the former $\rho N \quad(0<\rho<1)$ point of signal sequence $x(n)$ by $w_{L}(n)$ of a length $\rho N$ is weighted, a new sequence $x_{\rho N}(n)$ is achieved. Note that the value of $\rho$ can be adjusted according to the real need of harmonic analysis. Then, the spectrum of $x_{\rho N}(n)$ is obtained by using the FFT. For the new FFT sequence, the phase relationship between the $k$ th harmonic and the spectral line $X\left(\gamma_{k}^{\prime}\right)$ can be expressed as

$\phi_{k h}=\varphi_{\gamma_{k^{\prime}}}+\pi \rho\left(\gamma_{k}^{\prime}-\gamma_{k h}\right)$

where $X\left(\gamma_{k}^{\prime}\right)$ is the spectral line that is the nearest to the true peak position of the $k$ th harmonic in the new DFT sequence. $\varphi_{\gamma_{k^{\prime}}}$ and $\gamma_{k}^{\prime}$ are the initial phase and normalized frequency of spectral line $X\left(\gamma_{k}^{\prime}\right)$.

From Eq. (20) and Eq. (21), the phase difference between $X\left(\gamma_{k}\right)$ and $X\left(\gamma_{k}^{\prime}\right)$ is

$\Delta \varphi=-\pi(1-\rho) \Delta \varsigma_{k}+\pi \rho\left(\gamma_{k}^{\prime}-\gamma_{k}\right)$

Then, $\Delta \varsigma_{k}$ can be written as

$\Delta \varsigma_{k}=\frac{\beta}{\pi(1-\rho)}$

in Eq. (23), $\beta=\pi \rho\left(\gamma_{k}{ }^{\prime}-\gamma_{k}\right)-\Delta \varphi$.

From Eq. (23), the frequency $f_{i h}$ and phase $\phi_{k h}$ of the $k$ th harmonic are obtained, respectively 


$$
\begin{aligned}
f_{k h} & =\frac{\left(\gamma_{k}-\Delta \varsigma_{k}\right)}{T_{s} N} \\
\phi_{k h} & =\arctan \left(F_{\gamma_{k}} / Y_{\gamma_{k}}\right)+\pi \Delta \varsigma_{k}
\end{aligned}
$$

In Eq. (25), $F_{\gamma k}$ and $Y_{\gamma k}$ are the imaginary and real parts of spectral line $X\left(\gamma_{k}\right)$, respectively.

This is the procedure of estimating the harmonic parameters based on the presented method. The analysis is carried out on the $k^{\text {th }}$ harmonic. However, this method can actually be applied for calculating the parameters of any harmonic component.

In addition, when processing effort is under concern, from the calculation formulas of harmonic frequency and initial phase above, the presented method only needs to solve linear equation, so that the processing effort of the proposed method is almost the same as that of the analytical-solution interpolation method in [27]. In addition, when amplitude estimation is considered, the measurement of the proposed method is obtained from the FFT directly, without additional calculation procedure. Thus, the proposed method gives the better computation efficiency. Therefore, the proposed method mitigates the computation complexity greatly.

\section{Simulation and Implementation \\ 6.1 Harmonic parameters estimation under fundamental frequency Variation}

To demonstrate the applicability of the proposed algorithm, simulations are carried out by MATLAB. The signal model given in [11] is used for simulation, and the exact harmonic parameters are listed in Tab. 2.

$$
x(n)=\sum_{m=1}^{11} A_{m} \sin \left(2 \pi f_{m} n / f_{s}+\varphi_{m}\right)
$$

The sampling frequency is $2048 \mathrm{~Hz}$, and the total sampling number is 2500 .

The following methods are applied for the simulations, including the Hanning window based interpolated algorithm [11], the Blackman window based interpolated algorithm [19], and the window-length changeable phase difference correction algorithm with the improved $2^{\text {nd }}$-order FDMS-5FT convoluted window.

Since the fundamental amplitude is far larger than that of the other harmonics in power system, the second harmonics is subjected to the maximum influence. Therefore, the second harmonics is chosen to verify the precision of the presented algorithm. Figs. 6 and 7 exhibit the percent relative errors of the second harmonics amplitude and frequency. In simulation, the fundamental frequency is varying in the range from 49,5 to $50,5 \mathrm{~Hz}$. Besides, it is worth pointing out that the sampling numbers used in the proposed method with the improved $2^{\text {nd }}$-order FDMS-5FT convoluted window are 2300.

From Fig. 6 and 7, it is clearly shown that the accuracy of frequency estimation is enhanced by the presented method, compared with those of the methods based on the Hanning window and the Blackman window. Thus, it is confirmed that the presented method can availably overcome the interference resulting from the variation of the system frequency. As for the accuracy of amplitude, it can be seen that the amplitude measurement accuracy obtained by the improved FDMS-5FT convoluted window is also higher than those achieved by the Hanning window in [11] and the Blackman window given in [19].

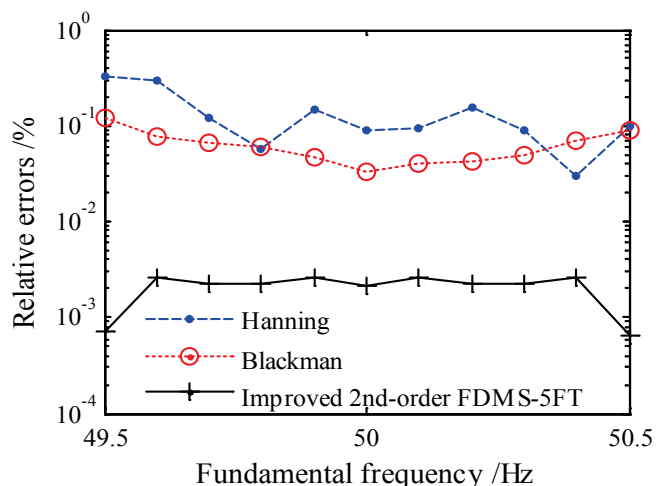

Figure 6 Relative errors of the second harmonic amplitude

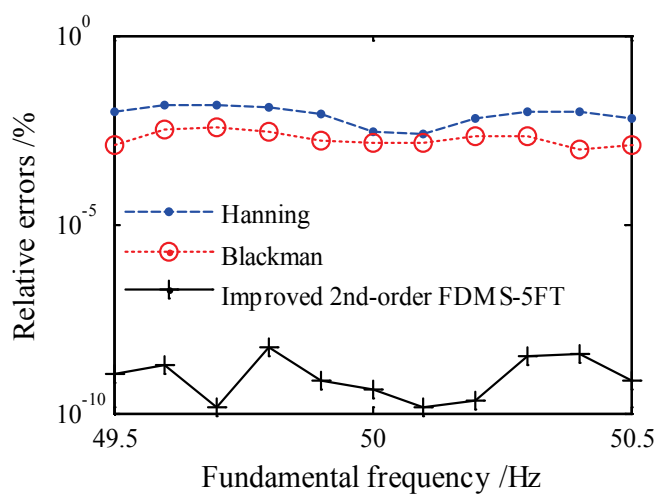

Figure 7 Relative errors of the second harmonics frequency

In addition, for different methods above applied for simulation ([11, 19], and the proposed method in turn), under the condition that harmonic analysis is performed only once, the computation time obtained by MATLAB with the same computer is $0,048 \mathrm{~s}, 0,058 \mathrm{~s}$, and $0,039 \mathrm{~s}$, respectively. It is clear that the computation time of the proposed method is the least. Therefore, the proposed method reduces processing efforts availably.

\subsection{Simulation with white noise}

The signal model in Eq. (26) is superposed with zeromean Gaussian noise, where the signal-to-noise ratios (SNR) vary from 10 to $100 \mathrm{~dB}$ at an increment of $10 \mathrm{~dB}$. The fundamental frequency is $49,5 \mathrm{~Hz}$. Under these conditions, the harmonic parameters are estimated by repeated measurements and the mean values of all the simulation results are then obtained.

As mentioned above, weak signal is easily affected by noise. Accordingly, the fourth harmonic is chosen for simulations to verify the Anti-noise performance of the proposed method.

Simulation results of the fourth harmonic amplitude/frequency are shown in Fig. 8 and 9, where the method based on the Hanning window in [11] and based on the 3-term 3 orders Nuttall window given in [28], and the proposed method with the improved $2^{\text {nd }}$-order FDMS- 
5FT convoluted window are used to make the comparison.

From Fig. 8 and 9, when SNR is less than $50 \mathrm{~dB}$, the influences of white noise to these different methods are serious, especially for the accuracy of harmonic amplitude. For SNR $>50 \mathrm{~dB}$, the accuracies obtained by these different methods are enhanced with the increase of SNR.
Particularly, the proposed method with the improved FDMS-5FT convoluted window gives the best accuracies. Accordingly, the proposed method has good anti-noise ability and can extract weak signal component precisely under noise.

\begin{tabular}{|c|c|c|c|c|c|c|c|c|c|}
\hline Harmonics & $1^{\mathrm{st}}$ & $2^{\text {nd }}$ & $3^{\text {rd }}$ & $4^{\text {th }}$ & $5^{\text {th }}$ & $6^{\text {th }}$ & $7^{\text {th }}$ & $9^{\text {th }}$ & $11^{\text {th }}$ \\
\hline$A_{m}(\mathrm{~V})$ & 240 & 0,1 & 12 & 0,1 & 2,7 & 0,05 & 2,1 & 0,3 & 0,6 \\
\hline$\theta_{m}\left({ }^{\circ}\right)$ & 0 & 10 & 20 & 30 & 40 & 50 & 60 & 80 & 100 \\
\hline
\end{tabular}

Table 3 Measurement results of voltages and phase of A-phase

\begin{tabular}{|l|c|c|c|c|c|c|}
\hline \multicolumn{1}{|c|}{ Harmonic order } & $1^{\text {st }}$ & $2^{\text {nd }}$ & $3^{\text {rd }}$ & $4^{\text {th }}$ & $5^{\text {th }}$ & $6^{\text {th }}$ \\
\hline Exact amplitudes $(\mathrm{V})$ & 220,362 & 1,16000 & 12,3730 & 1,02000 & 5,63000 & 0,537000 \\
\hline Relative errors of the proposed method $(\%)$ & 0,016 & $-0,197$ & $-0,021$ & 0,162 & $-0,037$ & 0,132 \\
\hline Exact phases $\left({ }^{\circ}\right)$ & 62,361 & 132,620 & 32,500 & 11,402 & 23,600 & 43,101 \\
\hline
\end{tabular}

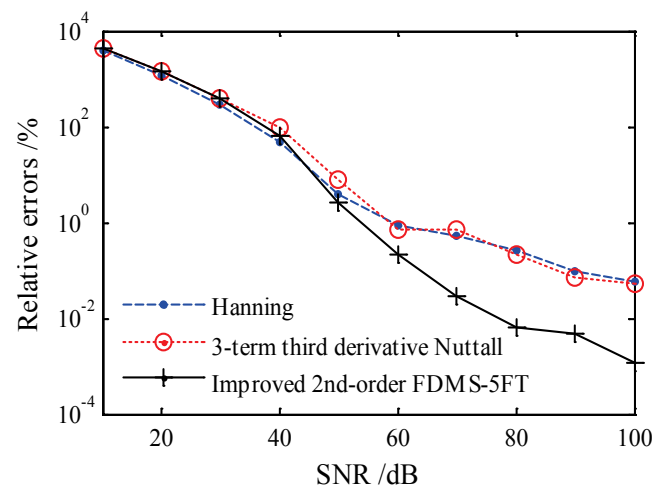

Figure 8 Relative errors of the fourth harmonic amplitude

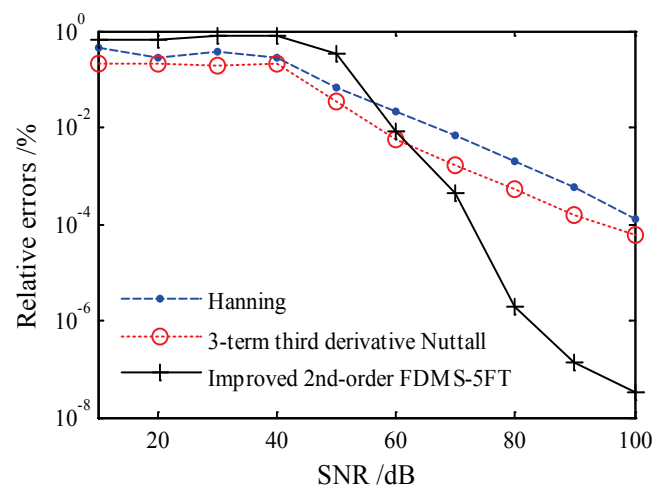

Figure 9 Relative errors of the fourth harmonic frequency

Finally, from the simulation results achieved in Fig. $6 \div 9$, we know that harmonic parameters, including evenorder harmonic and weak harmonic components, can be accurately evaluated by the proposed method under spectral leakage and white noise. Moreover, the improved FDMS-FT convoluted window based on window-length changeable phase difference correction algorithm is free from solving high-order equations. Thus, the proposed algorithm efficiently reduces the computation burdens.

\subsection{Implementation}

In practical engineering, the signals of power system are influenced not only by the variation of fundamental frequency or white noise but also by other kinds of interferences, such as quantization noise. Therefore, field test is necessary to verify the effectiveness of the method. The proposed method, in which the improved $2^{\text {nd }}$-order FDMS-5FT convoluted window is used for weighting, is tested by ARM-based embedded platform.

The main experiment devices include an ARM9 core based processor $\mathrm{S} 3 \mathrm{C} 2440$ with $16 \mathrm{~K}$ instruction cache, $16 \mathrm{~K}$ data cache, and a standard harmonic signal source. For the low resolution of the internal ADC of S3C2440, a high speed high resolution external $\mathrm{AD}$ converter ADS8364 is utilized. To meet the requirement of real time harmonic measurement, the processor main clock is set at $400 \mathrm{MHz}$, that is to say, the processor can run $400 \mathrm{M}$ instructions per second. In addition, standard harmonic signal is generated by Fluke 6100 A electrical power standard. For Fluke $6100 \mathrm{~A}$, the output resolutions of amplitude and frequency are $100 \mathrm{ppm}$ and $50 \mathrm{ppm}$ respectively, and phase resolution is 0,001 degree.

In experiment, the real harmonic parameters are given by Fluke $6100 \mathrm{~A}$ while the measured values are estimated by the ARM-based three-phase harmonic ammeter. The fundamental frequency is set to $50,1 \mathrm{~Hz}$, and the sample number is 1024 . The testing results of A-phase, which include the amplitude and initial phase of voltage, are listed in Tab. 3.

It is shown that the maximum relative errors of amplitude and phase based on the proposed method are $0,197 \%$ and $1,823 \%$, respectively. In addition, the measured value of the fundamental frequency was 50,096 $\mathrm{Hz}$, and the percent of relative error was $0,008 \%$. Thus, the testing results validate the correctness and effectiveness of the proposed method. Moreover, it is also clear that the proposed method can be implemented in embedded systems easily.

\section{Conclusions}

This paper focuses on the accurate and lowcomputation harmonic parameter estimation procedure. With this aim, a new type of flat-top windows is constructed, namely the improved FDMS-FT convoluted window. Then, a window-length changeable discrete phase difference correction method based on the new flattop window is presented for power harmonic parameter estimation. Compared with the classical flat-top window, the improved FDMS-FT convoluted window has the 
advantages of better sidelobe performance and flatter mainlobe, which can minimize the errors caused by spectral leakage and picket fence effect greatly. The proposed method has a simple calculation procedure, and the correction formulas of harmonic parameter only require solving linear equation, decreasing computational burden dramatically. In addition, the simulations and practical measurements indicate that without using the techniques of hardware synchronization, the proposed method can accurately estimate power harmonic parameter and be easily applied in embedded systems.

\section{Acknowledgements}

This work is supported by the National Natural Science Foundation of China (51577050) and the key project of science and technology of Wuxi Electric Power Company of China (5210301503RQ)

\section{References}

[1] Stojkov, M.; Trupinic, K.; Nikolovski, S. Procedure for determination of harmonic distortion along the distribution network. // Tehnicki Vjesnik-Technical Gazette. 16, 4(2009), pp. 19-26.

[2] Hani, V.; Alireza, A. Weighting method to identify interharmonics based on calculating the bandwidth in group-harmonics. // Journal of Power Electronics. 13, 1(2013), pp. 170-176. DOI: 10.6113/JPE.2013.13.1.170

[3] Jain, S. K.; Singh, S. N. Low-Order Dominant Harmonic Estimation Using Adaptive Wavelet Neural Network. // IEEE Transactions on Industrial Electronics. 61, 1(2014), pp. 3334-3342. DOI: 10.1109/TIE.2013.2242414

[4] Dalali, M.; Jalilian, A. Indices for measurement of harmonic distortion in power systems according to IEC 61000-4-7 standard . // IET Generation, Transmission \& Distribution. 9, 14(2015), pp. 1903-1912. DOI: 10.1049/ietgtd.2015.0366

[5] Tan, T. Y.; Chen, W. J.; Liu, K. P. Harmonic analysis based on time domain mutual-multiplication window. // Journal of Power Systems and Clean Energy. 4, 1(2016), pp. 47-53. DOI: 10.1007/s40565-016-0190-y

[6] Zeng, B.; Teng, Z. S. Parameter estimation of power system signals based on cosine self-convolution window with desirable side-Lobe behaviors. // IEEE Transactions on Power Delivery. 26, 1(2011), pp. 250-257. DOl: 10.1109/TPWRD.2010.2083701

[7] Chang, G. W.; Chen, C. J.; Liu, Y. J. Measuring power system harmonic and inter-harmonic by an improved fast Fourier transform based algorithm. // IET Generation, Transmission \& Distribution. 2, 2(2008), pp. 193-201. DOl: 10.1049/iet-gtd:20070205

[8] Orallo, C. M.; Carugati, I.; Maestri, S. Harmonics measurement with a modulated sliding discrete Fourier transform algorithm. // IEEE Transactions on Instrumentation and Measurement. 63, 4(2014), pp. 781793. DOI: $10.1109 /$ TIM.2013.2287801

[9] Qian, H.; Zhao, R. X.; Chen, T. Interharmonics analysis based on interpolating windowed FFT algorithm. // IEEE Transactions on Power Delivery. 22, 2(2007), pp. 10641069. DOI: 10.1109/TPWRD.2007.893187

[10] Reljin, I. S.; Reljin, B. D.; Papic, V. D. Extremely flat-top windows for harmonic analysis. // IEEE Transactions on Instrumentation and Measurement, 56, 3(2007), pp. 1025 1041. DOI: 10.1109/TIM.2007.894889

[11] Zhang, F.; Geng. Z.; Yuan, W. The algorithm of interpolating windowed FFT for harmonic analysis of electric power system. // IEEE Transactions on Power Delivery. 16, 2(2001), pp. 160-164. DOI: 10.1109/61.915476

[12] Agrez, D. Dynamics of frequency estimation in the frequency domain. // IEEE Transactions on Instrumentation and Measurement. 56, 6(2007), pp. 2111-2118. DOI: 10.1109/TIM.2007.908240

[13] Zeng, B.; Teng, T. Z.; Cai, C. Y. Harmonic phasor analysis based on improved FFT algorithm. // IEEE Transactions on Smart Grid. 2, 1(2011), pp. 51-59. DOl: 10.1109/TSG.2010.2078841

[14] Harris, F. J. On the use of windows for harmonic analysis with the discrete Fourier transform. // Proceedings of the IEEE. 66, 1(1978), pp. 51-83. DOl: 10.1109/PROC.1978.10837

[15] Nuttall, A. Some windows with very good sidelobe behavior. // IEEE Transactions on Acoustics, Speech, and Signal Process. 29, 1(1981), pp. 84-91. DOI: 10.1109/TASSP.1981.1163506

[16] Zhang, Z. J.; Liang, L. C.; Chen, C. Y. A new family of windows convolution windows and their applications. // Science in China, Series E: Technological Sciences. 35, 7(2005), pp. 773-784. DOI: 10.1360/03ye0531

[17] Wen, H.; Teng, Z. S.; Wang, Y. Spectral correction approach based on desirable sidelobe window for harmonic analysis of industrial power system. // IEEE Transactions on Industrial Electronics. 60, 3(2013), pp. 1001-1009. DOI: 10.1109/TIE.2012.2189531

[18] Song, S. P.; Ma, H. Z.; Xu, G. Power harmonic analysis based on 5-term maximum-sidelobe-decay window interpolation FFT method. // Automation of Electric Power Systems. 39, 22(2015), pp. 83-88.

[19] Wu, J.; Zhao, W. A simple interpolation algorithm for measuring multi-frequency signal based on DFT. // Measurement, 42, 2(2009), pp. 322-327. DOI: 10.1016/j.measurement.2008.06.008

[20] Daniel, B.; Dominique, D. Amplitude estimation by a multipoint interpolated DFT approach. // IEEE Transactions on Instrumentation and Measurement. IM-58, 5(2009), pp. 1316-1323. DOI: 10.1109/TIM.2009.2012950

[21] Wen, H.; Teng, T. Z.; Wang, Y. Simple interpolated FFT algorithm based on minimize sidelobe windows for powerharmonic analysis. // IEEE Transactions on Power Electronics. 26, 9(2011), pp. 2570-2579. DOI: 10.1109/TPEL.2011.2111388

[22] Salvatore, L.; Trotta, A. Flat-top windows for PWM waveform processing via DFT. // IEE Proceedings. 135, 6(1988), pp. 346-361.

[23] Ding, K.; Zhu, X. Y.; Xie, M. Synthesized correcting method of phase difference on discrete spectrum. // Journal of Vibrating Engineering. 15, 1(2002), pp. 114-118.

[24] Kusljevic, M. D.; Tomic, J. J.; Jovanovic, L. D. Frequency estimation of three-phase power system using weightedleast-square algorithm and adaptive FIR filtering. // IEEE Transactions on Instrumentation and Measurement. 59, 2(2010), pp. 322-329. DOI: 10.1109/TIM.2009.2023816

[25] Chen, K. F.; Li, Y. F. Combining the Hanning windowed interpolated FFT in both directions. // Computer Physics Communications. 178, 12(2008), pp. 924-928. DOI: 10.1016/j.cpc.2008.02.008

[26] Cortés, C. A.; Mombello, E.; Dib, R. A new class of flattop windows for exposure assessment in magnetic field measurements. // Signal Process. 87, 9(2007), pp. 21512164. DOI: $10.1016 /$ j.sigpro.2007.02.012

[27] Grandke, T. Interpolation algorithms for discrete Fourier transform of weighted signals. // IEEE Transactions on Instrumentation and Measurement. 32, 2(1983), pp. 350355. DOI: $10.1109 /$ TIM. 1983.4315077

[28] Belega, D.; Dallet, D. Multifrequency signal analysis by interpolated DFT method with maximum sidelobe decay 
windows. // Measurement. 42, 3(2009), pp. 420-426. DOI:

10.1016/j.measurement.2008.08.006

\section{Authors' addresses}

Shuping Song, PhD, Corresponding author

College of Energy and Electrical Engineering,

Hohai University, Nanjing 211100, China

E-mail: ssp17@126.com

\section{Hongzhong Ma, Professor}

College of Energy and Electrical Engineering,

Hohai University, Nanjing 211100, China

E-mail: hhumhz@163.com

\section{Zhiyan Zhang, Associate professor}

College of Electrical Information Engineering,

University of Light Industry Zhengzhou, 450002, China

E-mail:2004074@zzuli.edu.cn 